\title{
COMISSÃO NACIONAL DE RESIDÊNCIA EM ENFERMAGEM - CONARENF/COFEN: 15 ANOS DE HISTÓRIA
}

Julita Correa Feitosa ${ }^{1}$, Antônio Eduardo Vieira Santos ${ }^{2}$, Valderez Ribeiro de Andrade ${ }^{3}$, Rita Miyahara Kobayashi ${ }^{4}$, Ney da Costa Silva ${ }^{5}$

Objetivo: descrever marcos históricos, contribuições e relação da Comissão Nacional de Residência de Enfermagem (Conarenf) com a história da Residência de Enfermagem (RE) Brasileira. Metodologia: trata-se de um estudo descritivo, documental, retrospectivo aos últimos 15 anos de existência da Conarenf. Resultados: no estudo dos últimos 15 anos da história da Conarenf (2001-2015), os documentos legais e relatórios apontaram para: marcos históricos no credenciamento de programas de RE junto ao Conselho Federal de Enfermagem; sistemática de outorga dos títulos de especialista na modalidade de residência; consultoria e assessoria visando a qualidade desses programas; construção de espaços para discussão em eventos científicos. Conclusão: as contribuições da Conarenf foram determinantes para a regulamentação dos programas de residência em enfermagem no Brasil.

Descritores: Internato não Médico, Educação em Enfermagem, Educação de Pós-Graduação.

\section{NATIONAL COMMISSION FOR NURSING RESIDENCY - CONARENF / COFEN: 15 YEARS OF HISTORY}

Objective: to describe main landmarks, contributions and relationship between the Comissão Nacional de Residência em Enfermagem (Conarenf) with the history of Brazilian Nursing Residence (NR). Methodology: This is a descriptive, documental and retrospective study, related to the last fifteen years of Conarenf's existence. Results: In the study of the last fiftteen years of Conarenf's history (2001-2015), the legal documents and reports pointed to: historical landmarks in the NR Programs Accreditation by Federal Nursing Council; systematic for concession of specialist titles in residence category; consulting and counseling aimed at the quality of these programs; to creating places of discussion in scientific events. Conclusion: contributions of Conarenf was decisive to the regulation of Brazilian NR programs.

Descriptors: Non-medical Internship, Education in Nursing, Post- Graduation.

\section{COMISIÓN NACIONAL DE RESIDENCIA EN ENFERMERÍA - CONARENF / COFEN: 15 AÑOS DE HISTORIA}

Objectivo: describir los marcos históricos, contribuciones y relación de la Comisión Nacional de Residencia de Enfermería (Conarenf) con la historia de Residencia de Enfermería (RE) Brasileña. Metodología: Se trata de un estudio descriptivo, documental, retrospectivo de los últimos quince años del Conarenf. Resultados: En esto estudio de los últimos quince años de la historia de Conarenf (2001-2015), los documentos legales e informes señalaron para: momentos históricos en el proceso de acreditación de programas de RE por el Consejo Federal de Enfermería; sistemática de otorga de los títulos de especialista en residencia; consultoría y asesoría dirigidos a calidad de esos programas; construcción de espacios de discusión en eventos científicos. Conclusión: las contribuciones de Conarenf fueran determinantes para la reglamentación de programas de RE en Brasil.

Descriptores: Internado no Médico, Educación de Enfermería, Educación de Postgrado.

${ }^{1}$ Enfermeira. Especialista em Metodologia da Assistência de Enfermgem. Coordenadora da Comissão de Residência em Enfermagem- Conarenf/Conselho Federal de Enfermagem.

${ }^{2}$ Enfermeiro. Doutor em Saúde da Mulher e da Criança. Professor Adjunto da Faculdade de Enfermagem da Universidade Estadual do Rio de Janeiro.

${ }^{3}$ Enfermeira. Mestre em Saúde da Criança e do Adolescente. Gerente da Unidade de Formação e Educação em Saúde da Secretaria de Saúde do Recife,

Pernambuco.

${ }^{4}$ Enfermeira. Doutora em Enfermagem. Diretora do Serviço de Educação Continuada do instituto Dante Pazzanese de Cardiologia, São Paulo.

${ }^{5}$ Enfermeiro. Especialista em Geriatria e Gerontologia. Membro do Conaref/Cofen. 


\section{INTRODUÇÃO}

A primeira menção de especialização de profissionais de saúde com ênfase na prática profissional em serviço ocorreu em 1848, quando a Associação Médica Americana iniciou a formação de recursos humanos na área médica, privilegiando - ensino da prática clínica hospitalar e o adestramento profissional em serviço - a Residência Médica(1). Apresentava, como requisito, o profissional ter de morar na instituição onde o programa seria desenvolvido, visando estar à disposição do hospital em tempo integral.

Dentre outras profissões da área de saúde, há indícios de que a segunda profissão a adotar Programas de Especialização de seus profissionais na modalidade de Residência foi a enfermagem. Os programas de Residência em Enfermagem (RE) surgiram seguindo o modelo da Residência Médica (RM) brasileira, sendo o primeiro criado em 1961, no Hospital Infantil do Morumbi, filiado à então Legião Brasileira de Assistência, objetivando o aperfeiçoamento de enfermeiros na especialidade de Enfermagem Pediátrica. ${ }^{(2)}$

Os programas de RE se expandiram sem regulamentação e fiscalização e, em 1979, a Associação Brasileira de Enfermagem (ABEn) publicou recomendações originárias do Seminário sobre Residência(3) ${ }^{(3)}$ descrevendo a preocupação em caracterizar a residência como modalidade de curso de especialização, aprimorando conhecimentos teóricos e o desenvolvimento de pesquisa em enfermagem, mas com ênfase na prática(3)

$\mathrm{Na}$ década de 80 , os programas de RE mantiveramse sem regulamentação, até que, no início da década de 90, o Conselho Federal de Enfermagem (Cofen) e a ABEn retomaram os estudos sobre o assunto, visando configurar uma proposta de regulamentação dos programas.

Assim, em 1994, a Comissão Permanente de Educação da ABEn Nacional realizou uma oficina intitulada "RE no Brasil"(4). O documento originário dessa oficina apontava divergências quanto à duração e carga horária bem como críticas referentes à: priorização das questões de tecnologia e do trabalho; colocação das necessidades de saúde da população em segundo plano; aplicação de um modelo que não atendia às demandas da sociedade e nem causava impacto no perfil epidemiológico de saúde da população; objetivação de suprimento das deficiências da graduação e déficit de pessoal dos serviços de saúde ${ }^{(4)}$

Diante desse contexto, após o Seminário Nacional do Sistema Cofen/Conselhos Regionais, o Cofen emitiu documento final sobre a $\operatorname{RE}^{(5)}$ e, devido ao cenário de incertezas, constituiu um grupo de trabalho, visando propor uma regulamentação para os programas de RE. Dentre outros desdobramentos do trabalho realizado por esse grupo, foi constituída, no âmbito do Cofen, a Comissão Nacional de
Residência de Enfermagem (Conarenf).

Assim, na medida em que o resgate histórico do desenvolvimento dos Programas de Residência de Enfermagem no contexto da educação brasileira remete-nos à reflexão acerca da política da formação dos profissionais de saúde, sobre a legislação vigente e suas lacunas, bem como sobre a necessidade da educação permanente dos profissionais nos cenários de prática, preservando assim registros sobre sua evolução e consolidação no Brasil, o presente artigo objetivou descrever os principais marcos históricos e contribuições da Conarenf, e sua relação com a história da RE no Brasil.

\section{METODOLOGIA}

Trata-se de um estudo descritivo, documental, retrospectivo aos 15 anos de existência da Conarenf (20011015). Foi desenvolvido na sede do Cofen, onde ocorreram suas atividades, encontrando-se arquivados seus respectivos documentos. Para o desenvolvimento deste estudo, foram resgatadas atas de reuniões, relatórios anuais, programas de eventos científicos, projetos pedagógicos, manuais, legislações, processos administrativos e demais documentos pertencentes ao acervo da Conarenf.

A partir da leitura minuciosa e análise desses documentos, foram definidas categorias relacionadas ao contexto histórico da estruturação da Conarenf e suas principais contribuições, destacando-se o credenciamento dos programas de RE, a outorga de títulos de especialistas na modalidade de residência, a organização de eventos científicos, consultorias e assessorias.

\section{CONTEXTO HISTÓRICO DAESTRUTURAÇÃO DA CONARENF}

No início da década de 90, o Cofen propiciou espaços para o desenvolvimento e legitimação dos programas de RE no País, promovendo oficinas e seminários no território nacional, contando com profissionais experientes na coordenação e preceptoria dos programas de RE, residentes, representação das Instituições de Ensino Superior e demais órgãos de classe da categoria(6).

Desse período, destaca-se a realização do Seminário Nacional Cofen/Conselhos Regionais, em 1995, em Minas Gerais, em que o Anteprojeto de Lei( ${ }^{(7)}$ sobre a RE, resultante de discussões dos fóruns anteriores, foi avaliado, aprovado e encaminhado para tramitação nas instâncias competentes. Esse Anteprojeto(7) transformou-se no Projeto de Lei (PL) no 2.264/1996(8) que instituía a RE, como: "Curso de PósGraduação (Especialização) destinado às enfermeiras, sob a responsabilidade de uma Universidade, Instituição de Ensino Superior de Enfermagem ou Instituto de Pesquisa, público ou privado, caracterizado pelo aprofundamento do 
conhecimento científico e proficiência técnica decorrentes de treinamento em serviço, em regime de tempo integral".

Tratava-se de uma proposta consistente em seu conteúdo, que propunha um programa com a duração mínima de dois anos (3.800h). Entretanto, apesar do avanço de incluir a enfermagem e as outras profissões da área de saúde, durante sua tramitação, houve seu arquivamento(8).

É necessário enfatizar que o Cofen, paralelemente à tramitação do PL 2.264/96, iniciou um trabalho pioneiro e nacional para regulamentação da especialização de enfermeiros através de programas de RE, justificada pela inexistência de regulamentação emanada de esfera governamental para essa modalidade de ensino em serviço(b). Além disso, os programas de RE continuavam se estruturando em critérios próprios, de acordo com seus contextos particulares, com diferenças exorbitantes quanto à carga horária prática, teórica, repouso semanal, férias, dentre outros.

Em decorrência da forte pressão da categoria, particularmente dos residentes de enfermagem egressos desses programas, pela legitimação da qualificação profissional nessa modalidade, o Cofen constituiu um grupo de trabalho(6) composto por enfermeiros, docentes, representantes dos programas de RE dos diversos estados. $\mathrm{O}$ principal objetivo desse grupo foi estabelecer padrões mínimos para o registro do enfermeiro especialista na modalidade de RE, resultando na promulgação da Resolução COFEN 259/2001 ${ }^{(9)}$. Essa resolução estabeleceu os padrões mínimos para credenciamento de programas de RE em âmbito nacional e para a outorga de títulos de especialista na modalidade de residência, bem como procedeu encaminhamentos para criação da Conarenf, nomeada pela Portaria Cofen no 004/2002(10). Nos últimos 15 anos, a Conarenf foi composta por diferentes membros, conforme apresentado no Quadro 1.

Quadro 1: Composição da Conarenf entre 2006 a 2016. Brasília, 2016.

\begin{tabular}{|c|c|c|c|}
\hline Ano & Portaria & Composição & Marcos legais regulatórios no Cofen \\
\hline \multirow{6}{*}{2002} & \multirow{6}{*}{$\begin{array}{l}\text { Portaria Cofen no 004/2002, de } \\
\text { 11/03/2002. Designa membros para } \\
\text { Conarenf. }\end{array}$} & Dr. Edilson Sebastião Pimentel (RJ). & \multirow{6}{*}{$\begin{array}{l}\text { Resolução Cofen } 259 / 2001^{(10)} \\
\text { Decisão Cofen 064/2003(11) }\end{array}$} \\
\hline & & 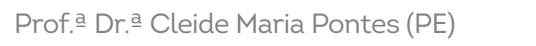 & \\
\hline & & Prof. Dr. Eliel de Oliveira Larrubia(RJ) & \\
\hline & & Prof. - Dr. - MárciaPitangaCavalcanti (SP) & \\
\hline & & Prof. a Dr. a Sônia Regina Pereira (SP) & \\
\hline & & Prof. a Dr. a Cristiane M.AmorinCosta (RJ) & \\
\hline \multirow{8}{*}{2006} & \multirow{8}{*}{$\begin{array}{l}\text { Portaria no 028/2006, de 29/05/2006. } \\
\text { Designa membros para Conarenf, revo- } \\
\text { gando a anterior. }\end{array}$} & Dr. Edilson Sebastião Pimentel(RJ). & \multirow{8}{*}{-} \\
\hline & & Prof. a Dr. a Cleide Maria Pontes (PE) & \\
\hline & & Prof. Dr. Eliel de OliveiraLarrubia(RJ) & \\
\hline & & Prof. D Dr. - MárciaPitangaCavalcanti (SP) & \\
\hline & & Dr. Ney da Costa Silva (RJ) & \\
\hline & & Prof. a Dr. a Sônia Regina Pereira (SP) & \\
\hline & & Prof. a Dr. a Cristiane M.AmorinCosta (RJ) & \\
\hline & & Sr. - Maria A. de Souza (RJ) - sec. adm. & \\
\hline \multirow{7}{*}{2007} & \multirow{7}{*}{$\begin{array}{l}\text { Portaria Cofen no 033/2007 de } \\
\text { 08/02/2007. Designa membros para } \\
\text { Conarenf, revogando a anterior. }\end{array}$} & Prof. Dr. Eliel de Oliveira Larrubia(RJ) & \multirow{7}{*}{-} \\
\hline & & Prof. a Dr. a Cleide Maria Pontes (PE) & \\
\hline & & Dr. Ney da Costa Silva (RJ) & \\
\hline & & Prof.a Dr. a Sônia Regina Pereira (SP) & \\
\hline & & Prof. a Dr. a Cristiane M.AmorinCosta (RJ) & \\
\hline & & Dr. Edilson Sebastião Pimentel (RJ) & \\
\hline & & Sr. ‥ Maria A de Souza (RJ) - sec. adm. & \\
\hline \multirow{7}{*}{2009} & \multirow{7}{*}{$\begin{array}{l}\text { Portaria Cofen no 400/2009, de } \\
\text { 29/07/2009. Designa membros para } \\
\text { Conarenf, revogando a anterior. }\end{array}$} & Prof. Dr. Eliel de Oliveira Larrubia(RJ) & \multirow{7}{*}{ - } \\
\hline & & Prof. a Dr. a Cleide Maria Pontes (PE) & \\
\hline & & Dr. a Julita Correia Feitosa (PE) & \\
\hline & & Prof. Dr. Do Sônia Regina Pereira (SP) & \\
\hline & & Prof. a Dr. a Cristiane M.AmorinCosta (RJ) & \\
\hline & & Dr. Edilson SebastiãoPimentel (RJ). & \\
\hline & & Sr. - Maria A de Souza (RJ)- sec.adm. & \\
\hline
\end{tabular}


Portaria Cofenno 307/2013, de

2013

17/04/2013. Designa membros para

Conarenf, revogando a anterior.

Portaria Cofen no $884 / 2014$, de

2014 29/08/2014. Designa membros para Conarenf, revogando a anterior.

Portaria Cofen no 556/2015. Designa

2015 membros para Conarenf, revogando a anterior
Prof. Dr. Eliel de Oliveira Larrubia(RJ)

Prof. a Dr. . Cleide Maria Pontes (PE)

Dr. a Julita CorreiaFeitosa (PE)

Dr. - Valderez Ribeiro de Andrade (PE)

Prof. - Dr. a Cristiane M. Amorin Costa (RJ)

Dr. - Julita CorreiaFeitosa (PE)

Dr. . Valderez Ribeiro de Andrade (PE)

Prof. Dr. Antonio Eduardo V. Santos (RJ)

Resolução Cofen 459/2014 (12)

Prof. a Dr. a Rika Miyahara Kobayashi (SP)

Prof. Dr. - Lissandra Martins Souza (DF)

Dr. a Julita CorreiaFeitosa (PE)

Dr. a Valderez Ribeiro de Andrade (PE)

Prof. Dr. Antonio Eduardo V. Santos (RJ)

Prof. a Dr. a Rika Miyahara Kobayashi (SP)

Dr. Ney da Costa Silva (RJ)

Fonte: elaborado pelos autores

Ao longo desse período, a Conarenf desenvolveu suas atividades de modo a consolidar as legislações e diretrizes, realizar diagnósticos dos programas de RE, capacitar os atores envolvidos no processo, bem como cadastrar e credenciar os programas de RE quando ainda não havia normativas próprias para esse modelo de formação, emanadas dos ministérios da Educação e da Saúde ${ }^{(6,9)}$.

\section{CONTRIBUIÇÕES DA CONARENF}

A Conarenf tem contribuido para a enfermagem brasileira por meio de diversas atividades, como credenciamento dos programas de RE e certificação de seus egressos; atualização na temática da RE, mediante a realização de eventos, além de atividades de assessoria e consultoria(11).

Através do credenciamento de programas de RE, a Conarenf buscava assegurar que obtivessem condições mínimas para seu adequado funcionamento e formação de especialistas, aos quais o Cofen viria a outorgar o título(6).

\section{Credenciamento dos Programas de Residência em Enfermagem}

$O$ credenciamento dos programas de RE junto ao Sistema Cofen/Conselhos Regionais conferia um padrão de qualidade respaldado em critérios de funcionamento dos serviços e da assistência à saúde dos usuários. Era realizado a partir da solicitação formal pela instituição responsável, mediante o encaminhamento do projeto político pedagógico e do preenchimento do formulário de credenciamento. Essa documentação era analisada pelos membros da Conarenf, emitido relatório e agendada visita técnica para fins de avaliação in loco e emissão de parecer final. Diante de um parecer favorável, o programa era credenciado e seus egressos fariam jus ao registro de título de especialista na modalidade de RE junto ao Cofen. Caso o parecer não fosse favorável, era estipulado prazo para as correções sugeridas e agendada nova avaliação(9).

Entre os anos de 2001 a 2010, a Conarenf assegurou o credenciamento de mais de trinta instituições responsáveis por setenta programas de RE, conforme listados no Quadro 2.

Quadro 2: Programas de RE credenciados junto ao Sistema Cofen/Conselhos Regionais, por Estado e Instituição.

Brasilia, 2016

\begin{tabular}{|c|c|c|}
\hline Estado & Instituição & Programa de Residência de Enfrmagem \\
\hline \multirow[t]{4}{*}{ Amapá } & Hospital de Clínicas Dr. Alberto Lima & 1. Enfermagem Cirúrgica \\
\hline & Hospital da Mulher Mãe Luzia & 2. Enfermagem Obstétrica \\
\hline & Hospital da Criança e do Adolescente & \multirow[t]{2}{*}{ 3. Saúde da Familia } \\
\hline & Hospital de Emergência Dr. Oswaldo Cruz & \\
\hline \multirow[t]{5}{*}{ Alagoas } & Marternidade Escola Santa Monica / Universidade de Ciências da Saúde & 1. Enfermagem Obstetrica \\
\hline & de Alagoas & 2. Enfermagem em Neonatologia \\
\hline & Serviço de Atendimento Móvel de Urgência - SAMU & 1. Enfermagem em Atendimento Pre Hospitalar \\
\hline & Universidade de Ciências da Saúde de Alagoas & 1. Enfermagem em Saúde Mental \\
\hline & & 2. Enfermagem em Infectologia \\
\hline Goiás & Associação de Combate ao Câncer em Goiás & 1. Enfermagem Oncológica \\
\hline São Paulo & Instituto Dante Pazzanese de Cardiologia & 1. Enfermagem Cardiovascular \\
\hline
\end{tabular}




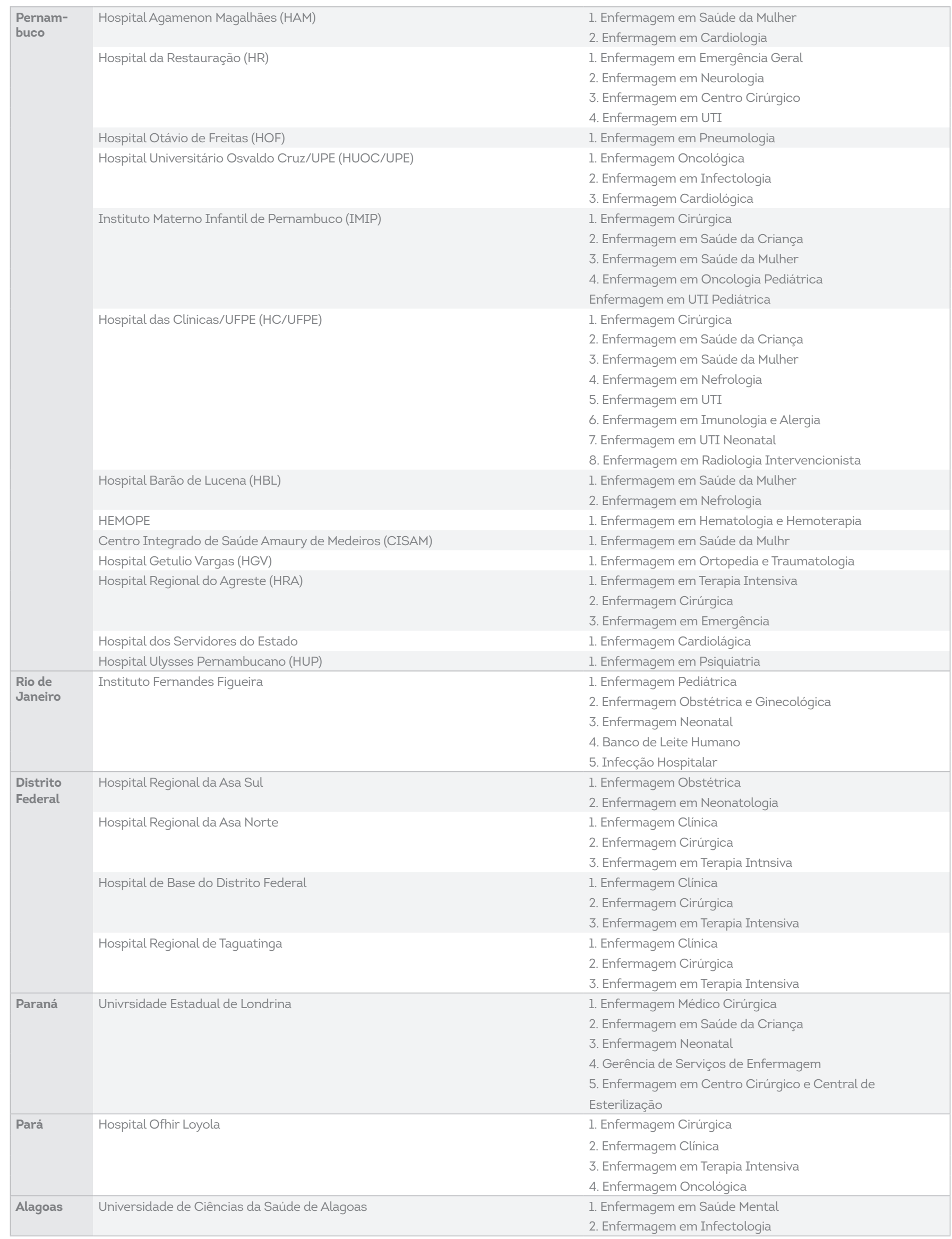

*fonte: Comissão Nacional de Residencia em nfermagm (Conarenf) / Cofen 
Contudo, a Conarenf deixou de realizar o credenciamento de programas de RE a partir de 2010, em decorrência das primeiras normativas regulatórias governamentais relacionadas aos programas de residência em saúde, emitidas pela Comissão Nacional de Residência Multiprofissional em Saúde (CNRMS), como a Lei no 11.129(13), no qual se instituiu a Residência em Área Profissional da Saúde, como sendo "a modalidade de ensino de Pós-graduação Lato sensu, voltada para a educação em serviço e destinada às categorias profissionais que integram a área de saúde, excetuada a médica".

A regulamentação desses programas de residência ocorreu a partir da constituição da CNRMS, como previsto no art. 14 dessa Lei. A CNRMS, criada em 2005, passaria a ser responsável por elaborar diretrizes, normas, propor e adotar medidas visando à qualificação e à consolidação de Programas de Residência(14).

\section{Outorga do Título de Especialistas na Modalidade Residência}

Datam de 2004 os primeiros registros da Conarenf sobre emissão de pareceres, relativos às solicitações de registro do título de especialista na modalidade de residência, aos egressos de programas credenciados por essa comissão, conforme constatado nos pareceres emitidos e que se encontram arquivados na sede do Cofen.

A partir da análise do banco de dados eletrônico da Conarenf, verificou-se que a sistemática de registro desse título de especialista constituída pela Conarenf e pelo Setor de Registro e Cadastro (SRC) no Cofen, objetivando a organização do processo de trabalho, das documentações, otimização de recursos, possibilitou maior agilidade no atendimento das solicitações emergenciais dos requerentes ao título.

O processo de registro de título de especialista na modalidade residência está estruturado em fluxograma, ilustrado na Figura 1.

Figura 1 - Fluxograma do Registro do Título de Especialista na modalidade de Residência. Brasília, 2015.

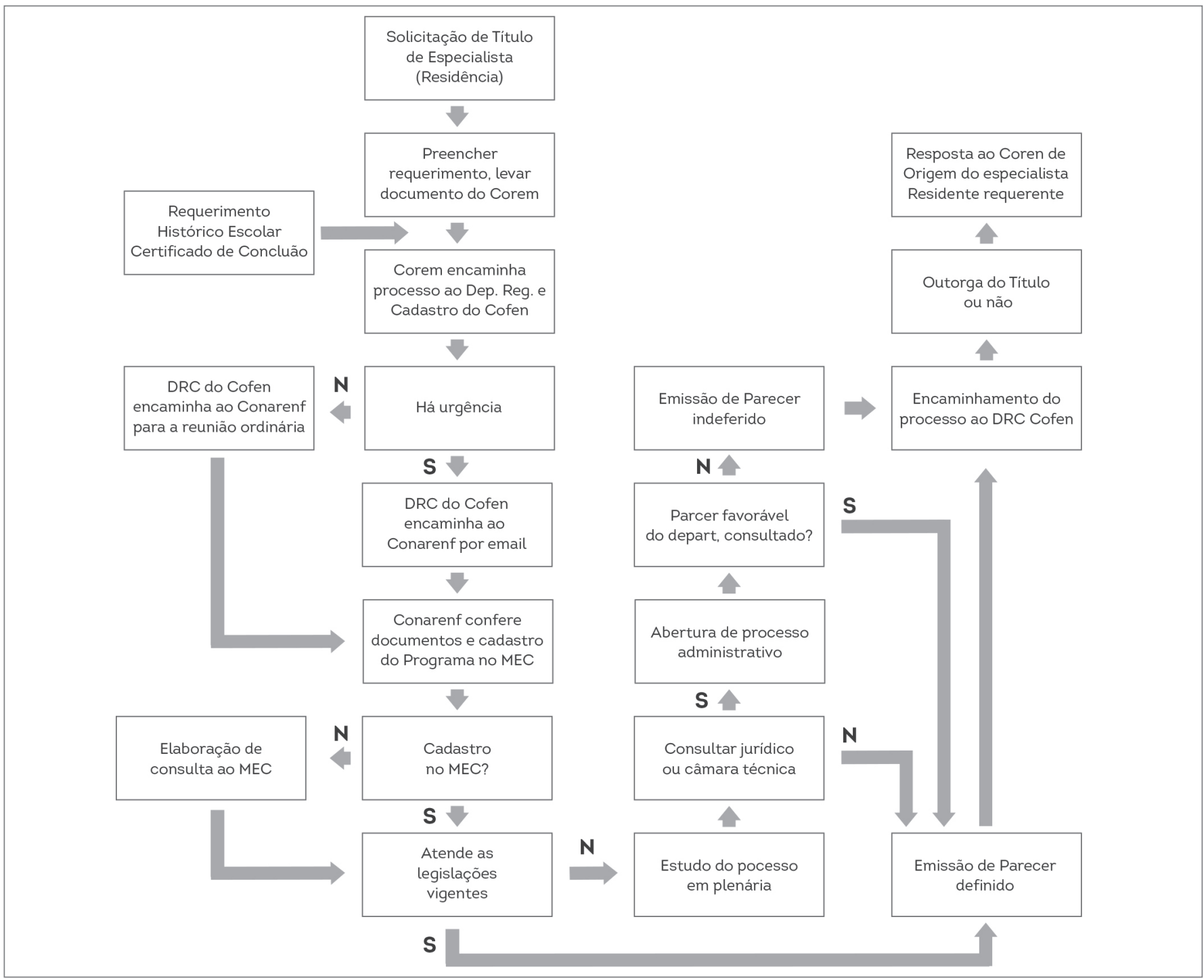


Destaque-se que os programas de RE cadastrados no Sistema da Comissão Nacional de Residencia Multiprofissional em Saúde (SisCNRMS) aguardam avaliação pela CNRMS para credenciamento e certificação. Uma vez que os egressos destes programas de residência solicitam o registro do título de especialistas, a Conarenfanalisa detalhadamente cada processo de solicitação de outorga de título de especialista na modalidade de residência remetida pelos Conselhos Regionais(1l).

Essa análise é realizada mediante conferência minuciosa da documentação enviada, à luz da regulação governamental vigente. Além disso, os programas de RE dos enfermeiros requerentes deverão estar cadastrados no SisCNRMS. No periodo de julho de 2014 a junho de 2016, foram analisados 390 processos de solicitação de registro de especialidade na modalidade de residência, conforme consta no banco de dados eletrônico da Conarenf.

\section{Organização de Eventos Científicos}

Desde sua criação, a Conarenf realizou eventos científicos periódicos e concomitantes ao Congresso Brasileiro dos Conselhos de Enfermagem (CBCENF), visando à atualização profissional e ao fortalecimento da produção e divulgação de conhecimentos específicos acerca da RE, sintetizado no Quadro 3.

Quadro 3 - Distribuição de eventos científicos, temas e local de realização, entre os anos de 2001 a 2015.

\begin{tabular}{|c|c|c|}
\hline Ano & Local & Tipo de evento e temáticas principais \\
\hline 2002 & Belo Horizonte & $\begin{array}{l}\text { Realizado o 1을 Congresso Nacional de Residências de Enfermagem durante o 7ㅇ CBCENF. } \\
\text { Divulgação e discussão sobre a Resolução Cofen 259/2001. }\end{array}$ \\
\hline 2004 & Fortaleza/CE & $\begin{array}{l}\text { Realizado o } 2 \text { Congresso Nacional de Residências de Enfermagem durante o } 8 \text { o CBCENF. } \\
\text { Processos de Implantação de Programas de RE e de credenciamento de programas de RE junto } \\
\text { ao Cofen. }\end{array}$ \\
\hline 2006 & Porto Seguro/BA & $\begin{array}{l}\text { Realizado o } 3 \text { o Congresso Nacional de Residências de Enfermagem durante o 9o CBCENF. } \\
\text { Desafios para a consolidação dos programas de RE credenciados pela Conarenf junto ao Cofen }\end{array}$ \\
\hline
\end{tabular}

$2007 \quad$ Curitiba/PR Realizado o 4으 Congresso Nacional de Residências de Enfermagem durante o 10으 CBCENF.

Promulgação da Portaria Interministerial MEC/MS 045/2007(16). Repercussão e perspectiva no cenário político e profissional da RE. Facilidades e dificuldades no desempenho específico dos preceptores e dos residentes.

$2008 \quad$ Belém/PA

$2009 \quad$ Belo Horizonte/MG
Realizado o 5으 Congresso Nacional de Residências de Enfermagem durante o 11으 CBCENF.

Implantação de Câmaras Técnicas na CNRMS. Processo de avaliação de RE e o impacto da produção cientifica no trabalho.

Realizado o 6으 Congresso Nacional de Residências de Enfermagem durante o 12으 CBCENF.

Relações entre as dimensões teórico e práticas: questões pedagógicas de métodos de ensino e avaliação por competências. Avaliação da Resolução Cofen 259/2001 para as questões da dimensão prática.

$2010 \quad$ Natal/RN

Realizado o 7으 Congresso Nacional de Residências de Enfermagem durante o 13으 CBCENF.

Dimensão ética dos programas de RE no sistema de saúde. Avaliação de impacto e do egresso da RE. Sistematização da Assistência de Enfermagem. Consolidação da produção do conhecimento, linhas de pesquisa para os programas de Residência, modalidades uni e multiprofissional.

$2010 \quad$ Natal/RN

Realizado o 7을 Congresso Nacional de Residências de Enfermagem durante o 13을 CBCENF.

Dimensão ética dos programas de RE no sistema de saúde. Avaliação de impacto e do egresso da RE. Sistematização da Assistência de Enfermagem. Consolidação da produção do conhecimento, linhas de pesquisa para os programas de Residência, modalidades uni e multiprofissional.

$2011 \quad$ Curitiba/PR

Realizado o 8으 Congresso Nacional de Residências de Enfermagem durante o 14으 CBCENF.

Projeto pedagógico. Perfil dos preceptores da Residência. Sistema de credenciamento, avaliação e monitoramento dos programas de RE realizada entre 2001 a 2009. 


\begin{abstract}
$2012 \quad$ Fortaleza/CE
Realizado o 9으 Congresso Nacional de Residências de Enfermagem durante o 15으 CBCENF.

Segurança do paciente. Indicadores de risco com os residentes de enfermagem. Estratégia de capacitação de preceptores da RE. Regulamentação de Titulação de especialistas na modalidade de RE. Estressores na atuação do residente de enfermagem e estratégia de minimização. A disciplina de ética na RE.
\end{abstract}

$2013 \quad$ Vitória/ES

Realizado o 10으 Congresso Nacional de Residências de Enfermagem durante o 16으 CBCENF

Ordenação na formação de recursos humanos na área de saúde e residência em enfermagem. Estruturação dos programas de residência em áreas prioritárias e os eixos norteadores revelados no processo de trabalho. Aplicação da tecnologia de informação e comunicação na sistematização da assistência e no cuidado de Enfermagem.

2014 Belém/PA Realizada palestra durante o 17으 CBCENF

"Aspectos históricos e legais dos programas de RE", com a Prof. a Dr. a Sonia Regina Pereira, representante do MEC, estimulando o trabalho colaborativo junto ao Cofen.

2015 João Pessoa/PB

$2016 \quad$ Cuiabá/MT Realizado o 1을 Encontro Nacional de RE durante o 18을CENF.

Processo de trabalho da Conarenf. Histórico dos programas de RE no Brasil e no mundo e os marcos regulatórios. Especificidades dos currículos de programas de RE no Brasil. Análise do Programa de RE Obstétrica.

Realizada mesa redonda durante o 190 CBCENF.

Avanços históricos e pedagógicos das residências de Enfermagem.

Fonte: Elaborado pelos autores

Mediante análise dos programas do Congresso Nacional de Residência de Enfermagem, no decorrer dos anos (2002 a 2012), as discussões avançaram em relação a questões legais, pedagógicas, diretrizes para os conteúdos programáticos mínimos, avaliação de egressos, produção de conhecimento científico e outras demandas que necessitavam de um olhar coletivo, para ampliação e solidificação da legitimidade dos programas de RE.

Outros temas relevantes permearam os eventos científicos da Conarenf, conforme análise da programação desses congressos, constando a discussão política da ordenação de formação de Recursos Humanos para o SUS, resgatando seus eixos norteadores, a residência como "padrão ouro" na formação dos profissionais de saúde e a estruturação de programas de RE nas áreas prioritárias.

Durante todos esses anos, a Conarenf, em seus eventos científicos, promoveu a discussão sobre a regulamentação de titulação de especialistas nessa modalidade, oportunizou condições para o empoderamento dos preceptores através de capacitação, tendo no último quinquênio, inovado sobre a aplicação da Tecnologia da Informação e Comunicação nos cenários diversificados da RE, conforme também constou nos programas do Congresso.

\section{Consultorias e Assessorias}

Em 2014, após revisão da Conarenf, foi incorporada, como sua atribuição, a consultoria e assessoria para implantação e monitoramento dos programas de RE. Assim, foram realizadas visitas às instituições executoras e prestada consultoria, com análise dos projetos pedagógicos, além de esclarecimento de dúvidas demandadas pelos coordenadores ${ }^{15}$.
A Conarenf tem desempenhado papel de destaque nas interfaces educativas interinstitucionais. Em 2015, realizou, além das consultorias e dos eventos científicos, a oficina de capacitação dos representantes do Cofen nas Câmaras Técnicas (CT) da CNRMS, objetivando: articular a profissão de Enfermagem junto ao MEC/MS, viabilizando o intercâmbio de informações; discutir o papel dos representantes das CT junto a CNRMS; e trabalhar com a nomenclatura dos cursos e a área de concentração na especificidade dos programas de RE, bem como participou do Programa de Capacitação de Avaliadores ${ }^{(15.16)}$

Atendendo às demandas de alguns programas de RE, a Conarenf realizou duas reuniões ordinárias, de forma descentralizada, aproximando-se dos Conselhos Regionais do Rio de Janeiro e de Pernambuco, permitindo conhecer a realidade dos programas dessas localidades e estabelecer parcerias, conforme previsto no planejamento e descrito nos relatórios anuais da Conarenf.

Considerando as mudanças no contexto nacional, com as publicações de resoluções da CNRMS, a Conarenf, em 2014, reformulou a Resolução Cofen 259/2001 ${ }^{(9)}$, sendo a mesma revogada pela Resolução Cofen 459/2014(12). A Conarenf foi demandada em 2015 a atualizar seu regimento, publicado na Decisão Cofen 064/2003(11), sendo o mesmo revogado pela Decisão Cofen 221/2015(18).

A partir dessas alterações regimentais, a Conarenf teve ampliado o seu escopo de atuação, sendo facultado à comissão:

- emitir pareceres da solicitação de outorga de registro de especialista na modalidade de residência, inclusive para egressos de programas na modalidade multiprofissional;- 
adotar diretrizes que contribuam ao aperfeiçoamento dos programas de RE;- atuar como articulador junto às Comissões/Técnicas análogas nos Conselhos Regionais;constituir-se como interlocutor da profissão de Enfermagem junto ao MEC/MS e às sociedades de especialistas;- deflagrar diretrizes nacionais reguladoras dos padrões de qualidade dos programas de RE;- atuar como instância consultora aos programas de RE, assessorando as instituições durante sua implantação;- deliberar sobre a realização de eventos científicos, culturais, estudos e pesquisas de interesse aos programas de RE; e divulgar estudos e atos normativos.

\section{CONCLUSÃO}

Este estudo descreveu as principais contribuições da Conarenf entre 2001 e 2015, consolidando a construção de uma sistemática de credenciamento de programas de RE e seu registro de especialista, como um primeiro passo para legitimação dessa modalidade de ensino no Brasil, quando ainda não havia regulamentação governamental própria.
Outra importante contribuição foi proporcionar espaços de discussão e construção coletiva formal e de atualização durante os Congressos Nacionais de Residência de Enfermagem promovidos junto aos CBCENF.

Avançando no tempo, e frente às necessidades das demandas do mercado de trabalho e do exercício da profissão, a Conarenf tem discutido sobre a temática da residência e seus respectivos títulos de especialidades, atualmente determinados na Portaria Cofen 389/2011(19).

Despontam como desafios atuais da Conarenf: propor diretrizes para determinação de conteúdos mínimos junto às sociedades de especialistas de enfermagem e a CNRMS; consolidar um trabalho no processo de avaliação do exercício profissional do enfermeiro residente junto aos setores de fiscalização dos Conselhos Regionais; realizar estudos para fixação de critérios de certificação de qualidade de programas de RE; e, estimular o trabalho interdisciplinar entre os diferentes conselhos profissionais estabelecendo competências comuns e especificas.

\section{REFERÊNCIAS}

1. Michel JLM, Oliveira RAB, Nunes MPT. Residência Médica no Brasil Cadernos da Associação Brasileira de Educação Médica. 2011 Out:(7):7-12.

2. Barros ALBL, Michel JLM. Curso de especialização em enfermagemmodalidade residência: experiência de implantação em um hospital-escola. Rev.latino-am.enfermagem,. 2000;8(1):5-11.

3. Associação Brasileira de Enfermagem. Recomendações do Seminário sobre a Residência em Enfermagem. Rev Bras Enferm 1979:32:121-5.

4. Associação Brasileira de Enfermagem. Documento da Oficina sobre "Diretrizes para a Residência em Enfermagem no Brasil". Salvador:ABEn;1994.

5. Conselho Federal de Enfermagem (BR). Documento final sobre Residência de Enfermagem. Rio de Janeiro:Cofen; 1994.

6. Larrubia, EO. Diretrizes legais para implantação: crescimento dos programas de residência em enfermagem no Brasil junto ao Cofen. Brasilia: Cofen; 2008.

7. Conselho Federal de Enfermagem (BR). Anteprojeto de Lei. Dispõe sobre a Residência em Enfermagem, e a sua respectiva Comissão Nacional. Rio de Janeiro;1996.

8. Câmara dos Deputados (BR). Projeto de Lei nㅇ 2.264/1996. Institui a Residência em Enfermagem e dá outras providências. Brasilia;1996.

9. Conselho Federal de Enfermagem (BR). Resolução 259/2001. Estabelece Padrões mínimos para registro de Enfermeiro Especialista, na modalidade de Residência em Enfermagem. Brasilia:Cofen;2001.

10. Conselho Federal de Enfermagem (BR). Portaria no 004/2002. Designa membros para Conarenf. Brasilia;Cofen; 2002.

11. Conselho Federal de Enfermagem (BR). Decisão 064/2003. Aprova o Regimento Interno da Conarenf. Brasilia:Cofen;2003.
12. Conselho Federal de Enfermagem (BR). Resolução no 0459/2014. Estabelece os requisitos mínimos para o registro de Enfermeiro Especialista. na modalidade de Residência em Enfermagem. Brasilia:Cofen; 2014.

13. Brasil. Lei no 11.129/2005. Institui o Programa Nacional de Inclusão de Jovens - Pro Jovem; cria o Conselho Nacional da Juventude - CNJ e a Secretaria Nacional de Juventude. Brasilia;2005.

14. Ministério da Educação /Ministério da Saúde. Portaria Interministerial MEC/MS no 1.320 de 11/11/2010. Dispõe sobre a estrutura, organização e funcionamento da Comissão Nacional de Residência Multiprofissional em Saúde - CNRMS. Brasilia: MEC/MS; 2015.

15. Conselho Federal de Enfermagem (BR). Decisão no 221/2015. Altera o Regimento Interno da Conarenf. Brasilia:Cofen;2015.

16. Ministério da Educação (BR). Secretaria de Educação Superior Diretoria de Desenvolvimento da Educação em Saúde. Coordenação Geral de Residências em Saúde. Comissão Nacional de Residência Multiprofissional em Saúde. Capacitação de Avaliadores da la Turma. Disponivel em: http://portal.mec.gov.br/index.php?option=com_ docman\&view=downloadEalias=43941-planilha-1-turma-capacitacaode-avaliadores-pdffcategory_slug=junho-2016-pdf\&Itemid=30192Acessado em: 15/02/2017

17. Conselho Federal de Enfermagem (BR). Portaria Cofen $\mathrm{n} \cong 1.052$ de 7 de outubro de 2014. Designação de colaboradores do Cofen para composição das Câmaras Técnicas da Comissão Nacional de Residência Multiprofissional em Saúde. Brasilia:Cofen;2014.

18. Conselho Federal de Enfermagem (BR). Decisão Cofen 221/2015. Altera o Regimento Interno da Conarenf. Brasilia:Cofen;2015.

19. Conselho Federal de Enfermagem (BR). Resolução no 389/2011. Atualiza no âmbito do Sistema Cofen/Conselhos Regionais de Enfermagem, os procedimentos para registro de título de Pós-Graduação Lato e Stricto Sensu concedido a enfermeiros e lista as especialidades. Brasilia:Cofen: 2011. 